\title{
Anti-Phosphorylated Histone H2AThr120: A Universal Microscopic Marker for Centromeric Chromatin of Mono- and Holocentric Plant Species
}

\author{
D. Demidov ${ }^{\text {a }} \quad$ V. Schubert ${ }^{\text {a }} \quad$ K. Kumke ${ }^{\text {a }} \quad$ O. Weiss ${ }^{\text {a }} \quad$ R. Karimi-Ashtiyani ${ }^{\mathrm{a}}$

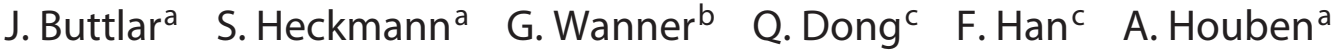 \\ ${ }^{a}$ Leibniz Institute of Plant Genetics and Crop Plant Research (IPK), Gatersleben, ${ }^{b}$ Ultrastructural Research, Department \\ Biology I, Biocenter, Ludwig Maximillian University Munich, Planegg-Martinsried, Germany; ' Institute of Genetics and \\ Developmental Biology, Chinese Academy of Sciences, Beijing, China
}

\section{Key Words}

CENH3 · Centromere - Drosera $\cdot$ Holokinetic · Meiosis · Mitosis

\begin{abstract}
Based on the analysis of 20 different monocot and eudicot species, we propose that the centromeric distribution of the phosphorylated histone H2AThr120 is evolutionary highly conserved across species with mono- and holocentric chromosomes. Therefore, antibodies recognizing the phosphorylated threonine 120 of the histone $\mathrm{H} 2 \mathrm{~A}$ can serve as a universal marker for the cytological detection of centromeres of mono- and holokinetic plant species. In addition, super resolution microscopy of signals specific to the centromere-specific histone $\mathrm{H} 3$ variant $\mathrm{CENH} 3$ and to $\mathrm{H} 2 \mathrm{AThr} 120$ ph revealed that these histone variants are incorporated into different nucleosomes, which form distinct, partly intermingled chromatin domains. This specific arrangement of both histone variants suggests different centromeric functions during the cell cycle.

(c) 2014 S. Karger AG, Basel
\end{abstract}

\section{KARGER}

E-Mail karger@karger.com

www.karger.com $/ \mathrm{cgr}$
The centromere of monocentric chromosomes is morphologically recognizable as a constriction during the mitotic metaphase. However, due to the small size of the chromosomes or a holocentric chromosome structure, the microscopical detection of centromeres is impaired in some species. Also, in contrast to telomeres, centromeres are not specified by highly conserved DNA sequences, and therefore, centromere detection by in situ hybridization is only possible if centromere-specific probes are available. Contrary to centromeric DNA, structural and functional kinetochore proteins are highly conserved between species [Houben and Schubert, 2003].

Human CREST antisera from patients with a variant of scleroderma have been used to label animal kinetochores [Moroi et al., 1980]. Two CREST sera were the first markers to identify kinetochores of some plant species [Mole-Bajer et al., 1990; Houben et al., 1995]. More recently, besides other kinetochore-specific antibodies (e.g. anti-CENPC [Dawe et al., 1999], anti-MAD2 [Yu and Dawe, 2000], CENPE, and SKP1 [ten Hoopen et al., 2000]), the evolutionarily conserved centromere-specific histone H3-variant CENH3 (in humans called CENPA) 
has become a popular centromere-specific marker. CENH3 determines the chromosomal position of kinetochore assembly and forms a link between the centromeric DNA and the proteinaceous kinetochore in eukaryotes [Blower et al., 2002]. Anti-CENH3 antibodies were frequently used for various centromere-related studies in plants [Nagaki et al., 2004]. Dependent on the epitope used for the generation of the antibody, some of the $\mathrm{CENH} 3$ antibodies cross-react with $\mathrm{CENH} 3$ of related species (e.g. anti rice-CENH3 [Nagaki et al., 2004] recognizes Aegilops markgrafii [Schubert, 2010], Coix lacrymajobi [Han et al., 2010], maize [Jin et al., 2004], sugarcane [Nagaki and Murata, 2005], wheat [Liu et al., 2008; Zhang et al., 2010], barley [Nasuda et al., 2005; Houben et al., 2007; Zhang and Dawe, 2012], and rye [Houben et al., 2011] or only the species used for the generation of the antibody e.g. A. thaliana [Talbert et al., 2002] and barley [Sanei et al., 2011]). However, no universal plant centromere-specific marker has been reported so far. Only antibodies specific for post-translational modifications of histones, namely phosphorylated histone $\mathrm{H} 3$ at residues serine 10 [Houben et al., 1999] and serine 28 [Gernand et al., 2003], were used as a marker for active mitotic pericentromeres in a wide range of plant species.

Another cell-cycle dependent histone mark involved in centromere function has been identified recently. Phosphorylated histone $\mathrm{H} 2 \mathrm{~A}$ at serine 121 in fission yeast (equivalent to human $\mathrm{H} 2 \mathrm{~A}$ Thr120) promotes the recruitment of shugoshin, a protein which protects cohesin at centromeres from cleavage by separase during mitosis and meiosis [Kawashima et al., 2010; Wang et al., 2011; Gutierrez-Caballero et al., 2012]. The conserved spindle assembly checkpoint protein kinase Bub1 (budding uninhibited by benzimidazole 1) phosphorylates $\mathrm{H} 2 \mathrm{~A}$ at this position [Kawashima et al., 2010].

Dong and Han [2012] identified that some members of the $\mathrm{H} 2 \mathrm{~A}$ gene family of maize contain a threonine residue 133 in the evolutionarily conserved LLPKKT/Sregion that undergoes phosphorylation in a cell cycledependent manner. During mitosis and meiosis, H2AThr133 phosphorylation in maize (equivalent to human H2AThr120) is enriched in metaphase specifically at centromeric regions. Then it decreases during late anaphase and telophase. Throughout meiosis, phosphorylation of $\mathrm{H} 2 \mathrm{~A}$ at Thr 133 persists at the centromeric regions. Immunostaining of a dicentric maize chromosome revealed that only the active centromere is marked by this histone modification. Also, cross-reactivity of this antibody with the centromeres of wheat and rice was reported [Dong and Han, 2012].

A Universal Centromere Marker
In the present study we demonstrate that antibodies recognizing the phosphorylated threonine 120 of histone $\mathrm{H} 2 \mathrm{~A}$ in humans can serve as a universal marker for the cytological detection of centromeres in mono- and polycentric plant species. In addition, we show that CENH3 and H2AThr120ph are components of different nucleosomes composing distinct intermingled centromeric chromatin domains.

\section{Materials and Methods}

Plant Material

The following plant species have been used: eudicots: Arabidopsis thaliana, Vicia faba (field bean), Lens culinaris (lentil), Gossypium hirsutum (cotton), Daucus carota (carrot), Beta vulgaris (sugar beet), Brachycome dichromosomatica, Solanum lycopersicum (tomato), Nicotiana tabacum (tobacco), and the sundew species Drosera binata, D. alicia, and D. rotundifolia; monocots: Zingeria biebersteiniana, Luzula luzuloides, L. elegans, Secale cereale (rye), Hordeum vulgare (barley), Triticum aestivum subsp. spelta (dinkel wheat), Allium fistulosum (Welsh onion), and Cyperus alternifolius (umbrella papyrus).

\section{Chromosome Preparation and Indirect Immunofluorescence}

For immunodetection, root tips from all species, staged anthers from $S$. cereale, and flower buds from A. thaliana were isolated and fixed in $4 \%$ paraformaldehyde in phosphate buffered saline (PBS) as previously described [Manzanero et al., 2000]. S. cereale, H. vulgare, and $V$. faba root tips were digested with $2 \%$ pectinase, $2 \%$ cellulase 'Onozuka R-10', and 2\% pectoylase Y-23, and squashes were made in PBS. To avoid non-specific antibody-binding, slides were blocked for $1 \mathrm{~h}$ in $4 \%$ BSA plus $0.1 \%$ Triton X-100 in PBS at room temperature, prior to $2 \times 5$ min washes in PBS, and incubated with the primary antibodies in a humid chamber for $12 \mathrm{~h}$ at $4{ }^{\circ} \mathrm{C}$. The following primary antibodies were used: rabbit antiphosphorylated histone H2AThr120 (Abcam, ab111492, diluted 1: 200), guinea pig anti-barley aCENH3 (diluted 1:100 [Sanei et al., 2011]), and monoclonal mouse anti-H3Ser10ph (Abcam, ab14955, diluted 1:1,000). After washing for $15 \mathrm{~min}$ in PBS, the slides were incubated in rhodamine-conjugated anti-rabbit IgG (Dianova) and FITC-conjugated anti-mouse IgG (Dianova), diluted 1:200 in PBS, $3 \%$ BSA for $1 \mathrm{~h}$ at $37^{\circ} \mathrm{C}$. After final washes in PBS, the preparations were counterstained with 4,6-diamidino-2-phenylindole hydrochloride (DAPI) in antifade. The slides were analyzed with an Olympus BX61 microscope equipped with an ORCA-ER CCD camera. Deconvolution microscopy was employed to reduce outof-focus blur. To achieve an optical resolution of ca. $100 \mathrm{~nm}$, we applied structured illumination microscopy using a C-Apo $63 \mathrm{x} / 1.2 \mathrm{~W}$ Korr objective of an Elyra microscope system and the software ZEN (Zeiss).

\section{Phosphatase Treatment of Meristematic Cells}

To demonstrate phospho-specificity of the H2AThr120phantibody, rye chromosomes were incubated with a mix of 100 units alkaline phosphatase (Sigma-Aldrich) and 4,000 units lambda protein phosphatase (NEB) in $100 \mu \mathrm{l}$ lambda phosphatase buffer (50 mM HEPES, $100 \mathrm{~mm} \mathrm{NaCl}, 2 \mathrm{~mm}$ DTT, $1 \mathrm{mM} \mathrm{MnCl}_{2}, 0.01 \%$ 
Brij-35, $\mathrm{pH} 7.5$ ) at $30^{\circ} \mathrm{C}$ overnight. Slides were then washed $3 \times 5$ min in $1 \times$ PBS and processed for immunofluorescence as described above.

Chromosome Preparation and Immunogold Labeling for Scanning Electron Microscopy

T. aestivum chromosomes were prepared for scanning electron microscopy (SEM) as described by Schubert et al. [1993]. Briefly, root tips were fixed with $2 \%$ formaldehyde (in $10 \mathrm{mM}$ Tris, $10 \mathrm{~mm}$ $\mathrm{Na}_{2}$ EDTA, $100 \mathrm{~mm} \mathrm{NaCl}$ ), mechanically dispersed (Polytron ${ }^{\circledR}$ $5 \mathrm{~mm}$ mixer, Kinematica, Switzerland) in isolation buffer (15 mM Tris, $2 \mathrm{mM} \mathrm{Na} 2$ EDTA, $0.5 \mathrm{mM}$ Spermin, $80 \mathrm{mM} \mathrm{KCl,} 20 \mathrm{~mm}$ $\mathrm{NaCl}, 15 \mathrm{~mm}$ Mercaptoethanol, 1\% Triton-X-100), and spun ('swing out' centrifugation) onto carbon coated glass slides. Specimens were washed in PBS buffer, blocked in $0.1 \%$ bovine serum albumin $+0.1 \%$ Tween 20 in PBS for $30 \mathrm{~min}$, and incubated with the H2AThr120ph antibody (1:100 diluted in blocking solution) for $2 \mathrm{~h}$. After washing, specimens were incubated with anti-rabbitAlexa488-Nanogold ${ }^{\circledR}$ (Nanoprobes) for $2 \mathrm{~h}$. Specimens were washed and post-fixed with $2.5 \%$ glutaraldehyde in PBS and subsequently silver enhanced (HQ Silver, Nanoprobes) according to the manufacturer's instructions. Specimens were washed in PBS, followed by distilled water, dehydrated in acetone, critical point dried from $\mathrm{CO}_{2}$, cut to size, and mounted onto aluminum stubs. Specimens were examined uncoated with a Zeiss Auriga CrossBeam Workstation. Micrographs were taken at accelerating voltages from $1 \mathrm{kV}$ (SE-signal; chamber SE-detector) and $30 \mathrm{kV}$ (BSEsignal; chamber QBSD-semiconductor detector).

\section{Protein Extraction and Western Blot Analysis}

Nuclear proteins from $200 \mathrm{mg}$ of flower buds and leaves of A. thaliana were isolated according to Gendrel et al. [2005]. A total of $10 \mu \mathrm{g}$ of protein was separated on 15\% SDS/PAGE gels and subsequently blotted on polyvinylidene fluoride membranes. Membranes were incubated first with primary antibodies (antihistone H3, ab1791, Abcam, diluted 1:2,500; anti-H2AThr120ph, ab111492, Abcam, diluted 1:2,000) and then with a secondary antibody (anti-rabbit IgG: IRDye800CW conjugated, LI-COR, diluted 1:5,000). Fluorescence signals were detected by a LI-COR Odyssey imaging system.

\section{Results and Discussion}

To test whether the amino acid sequence of human histone $\mathrm{H} 2 \mathrm{~A}$ flanking the phosphorylation site of threonine 120 is evolutionarily conserved, a sequence comparison was performed with the deduced protein sequence of histone $\mathrm{H} 2 \mathrm{~A}$ genes of 17 different lower and higher plant species. The comparison revealed that a large proportion of the $\mathrm{H} 2 \mathrm{~A}$ region used for the generation of the H2AThr120ph-specific antibody is conserved across species (online suppl. fig. 1; for all online suppl. material, see www.karger.com/doi/10.1159/000360018). However, in some species, threonine 120 of histone H2A is replaced by serine. Serine is the most common replace- ment for threonine which is also capable of being phosphorylated by kinases [Betts and Russell, 2007]. We would like to note that the histone H2A family in many eukaryotes typically contains different isoforms with either threonine or serine at this position. Further, anti$\mathrm{H} 2 \mathrm{AThr} 120 \mathrm{ph}$ recognized a protein of the histone $\mathrm{H} 2 \mathrm{~A}-$ type, molecular mass of $\sim 15 \mathrm{kDa}$ on Western blots, of $A$. thaliana nuclear protein extracts (online suppl. fig. 2). Since the histone H2A amino acid sequences of many plants are highly similar, the antibody employed is likely able to recognize histone $\mathrm{H} 2 \mathrm{AThr} 120 \mathrm{ph}$ modification in different species.

The localization of an anti-H2AThr120ph epitope during the cell cycle of $A$. thaliana was analyzed by indirect immunofluorescence. Mitotic chromosomes revealed centromeric signals with the onset of chromosome condensation (fig. 1A). To confirm that the antibody recognizes the phosphate moiety on the Thr120-residue of $\mathrm{H} 2 \mathrm{~A}$, we treated fixed rye root tips with phosphatases followed by incubation with the antibodies. Absence of specific immunofluorescence signals demonstrated that only the phosphorylated variant of $\mathrm{H} 2 \mathrm{~A}$ is recognized by antiH2AThr120ph. In untreated controls, strong immunostaining signals were detected at all centromeres (online suppl. fig. 3).

To see whether the position of H2AThr 120ph and that of the centromeric histone $\mathrm{H} 3$ variant (CENH3) coincide as suggested by Dong and Han [2012], the large somatic metaphase chromosomes of $H$. vulgare were analyzed. Double labeling with anti- $\alpha \mathrm{CENH} 3$ and antiH2AThr120ph revealed the localization of both histones in the primary constriction of all chromosomes. Consistent with the proposed function of H2AThr120ph as an interactor of the sister chromatid cohesion protector sughoshin [Kawashima et al., 2010; Wang et al., 2011; Gutierrez-Caballero et al., 2012], phosphorylated H2A localized more towards the inner domain of the centromere. CENH3 appeared mainly at the periphery of the sister chromatids. To ascertain the detailed arrangement of both centromere components, we applied structured illumination microscopy to achieve a resolution below the limit of light microscopy ( 100 nm) (fig. 2; online suppl. video 1). Both immunosignals form distinct, partly intermingling structures. This suggests that CENH3 and H2AThr120ph are components of different nucleosomes forming chromatin subdomains which localize close to each other. These subdomains are likely responsible for different centromeric functions, namely centromere formation and control of centromere cohesion. 


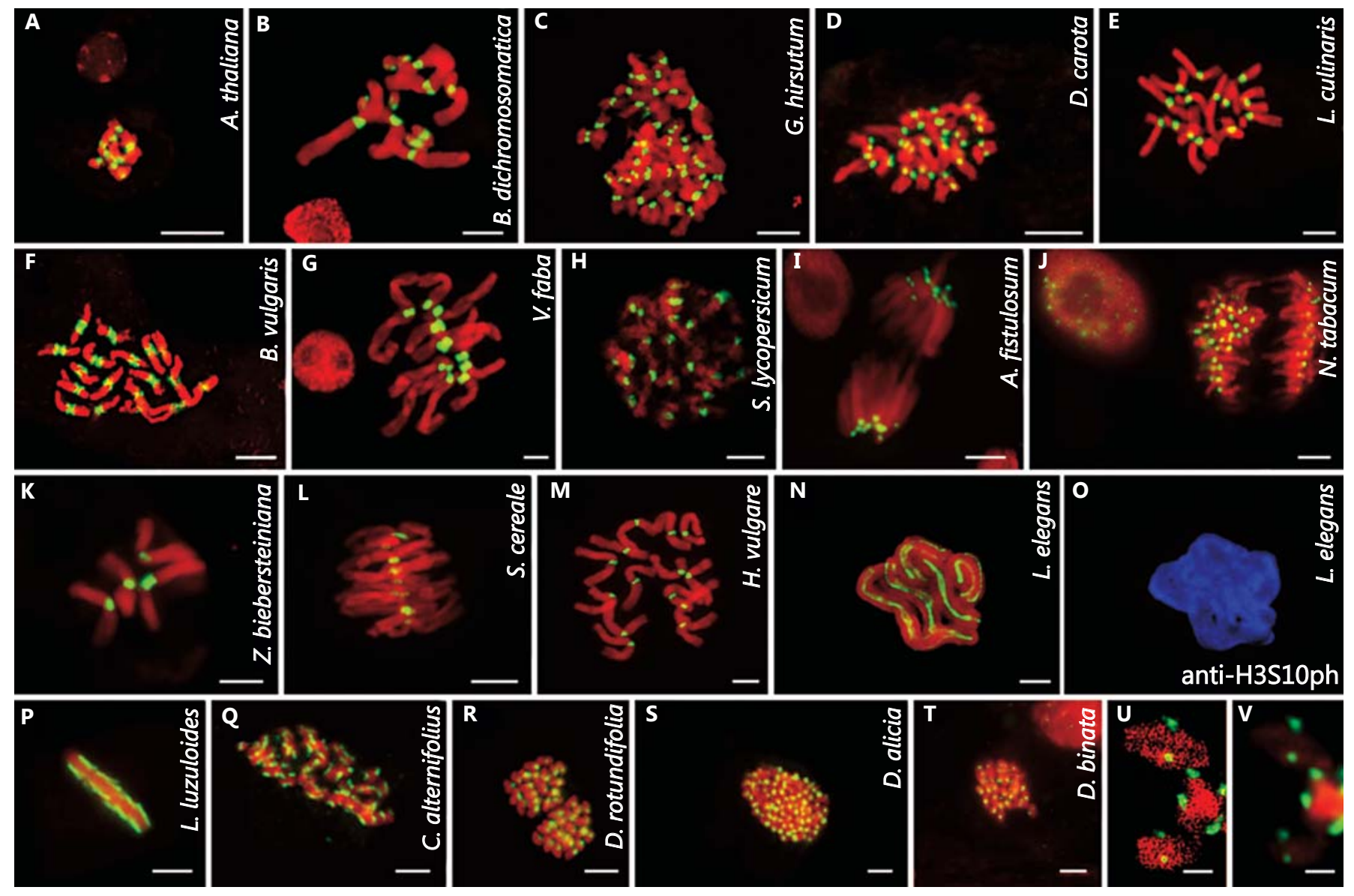

Fig. 1. Anti-histone H2AThr120ph recognizes centromeres of mono- and holocentric metaphase chromosomes of mono- and eudicot species. Fluorescent lightmicrographs of A Arabidopsis thaliana, B Brachycome dichromosomatica, C Gossypium hirsutum, D Daucus carota, E Lens culinaris, F Beta vulgaris, G Vicia faba, H Solanum lycopersicum, I Allium fistulosum, J Nicotiana tabacum, $\mathbf{K}$ Zingeria biebersteiniana, L Secale cereale, $\mathbf{M}$ Hordeum vulgare, $\mathbf{N}$ Luzula elegans, P Luzula luzuloides, Q Cyperus alternifolius, $\mathbf{R}$ Drosera rotundifolia, $\mathbf{S}$ Drosera alicia, and $\mathbf{T}-\mathbf{V}$ Drosera binata mitotic cells after immunostaining with antibodies recognizing the phosphorylated threonine 120 of histone H2A (in yellow). Only L. elegans chromosomes were additionally labeled with antiH3Ser10ph (in blue) (0). To demonstrate the monocentric-like distribution of H2AThr120ph signals, selected chromosomes of $D$. binata in $\mathbf{U}$ and $\mathbf{V}$ were visualized by structural illumination microscopy and epifluorescence microscopy, respectively. Size bars represent $5 \mu \mathrm{m}$ in $\mathbf{A}-\mathbf{T}$ and $1 \mu \mathrm{m}$ in $\mathbf{U}$ and $\mathbf{V}$.
Adequate structural preservation of mitotic chromosomes in combination with immunogold labeling allowed a comparative investigation of the distribution of phosphorylated histone H2AThr120 with high resolution SEM. T. aestivum metaphase chromosomes are ultrastructurally characterized by densely-packed chromomeres and a centromeric region characterized by fewer chromomeres and parallel fibrils (fig. 3A). Intense H2AThr120ph-specific signals were evenly distributed over the primary constriction (fig. 3B). Weak immunosignals were found along the chromosome arms. Distribution of CENH3 labels previously analyzed by SEM re- vealed a comparable distribution in the primary constriction [Houben et al., 2007]. In contrast, pericentromerespecific histone $\mathrm{H} 3$ modification $\mathrm{H} 3 \mathrm{Ser} 10 \mathrm{ph}$, although also distributed along the chromosome arms, exhibited a maximum density in the pericentric regions and a minimum, namely a signal gap, at the primary constriction [Schroeder-Reiter et al., 2003].

Anti-phosphorylated histone H2AThr 120 cross-reacts with the centromeric chromatin of mono- and holocentric chromosome species. To test whether the antibody recognizing phosphorylated H2AThr120 can serve as a universal maker for the cytological detection of plant centro- 


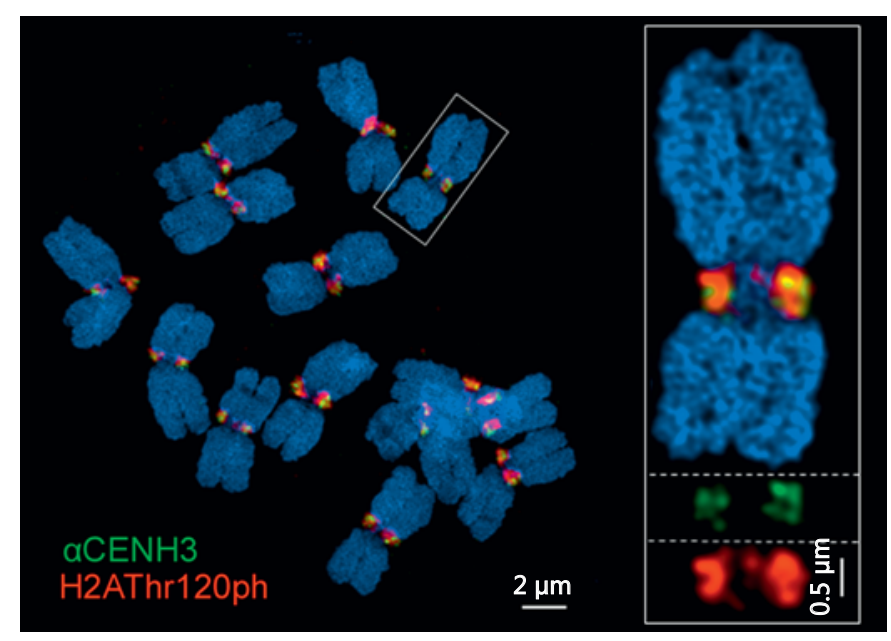

Fig. 2. Structured illumination microscopy resolves the arrangement of aCENH3 and H2AThr120ph at barley centromeres. Both histone variants are incorporated into different nucleosomes which form distinct, partly intermingling chromatin structures. The inset shows a further enlarged chromosome (top) and individual immunosignals (below) (see also online suppl. video 1).

meres, we immunolabeled diverse mono- and eudicot species possessing monocentric chromosomes with different genome sizes. Anti-H2AThr120-specific signals colocalized with the position of the centromere of mitotic chromosomes in all 13 species analyzed (fig. 1A-M). In addition, in a number of species with monocentric chromosomes (tomato, barley, rye, field bean, tobacco) centromeric signals were found in meristematic interphase nuclei. Also, rye chromosomes displayed phosphorylated H2AThr 120 at centromeres during meiosis I and II (fig. 4).

To investigate whether the type of centromere affects the distribution of phosphorylated $\mathrm{H} 2 \mathrm{~A}$, mitotic chromosomes of the holocentric species $L$. luzuloides and $L$. elegans were investigated. Both Juncaceae species show a continuous distribution of CENH3 along almost the entire length of mitotic metaphase chromosomes [Nagaki et al., 2005; Heckmann et al., 2011]. A similar centromerelike distribution was found for anti-H2AThr120ph at metaphase (fig. $1 \mathrm{~N}, \mathrm{P}$ ). In contrast, the pericentromerespecific mark H3Ser10ph revealed a uniform labeling of the condensed mitotic chromosomes (fig. 1O) as previously demonstrated by Gernand et al. [2003]. This implies that the centromeric distribution of phosphorylated H2AThr120 is evolutionary conserved across species with mono- or holocentric chromosomes.

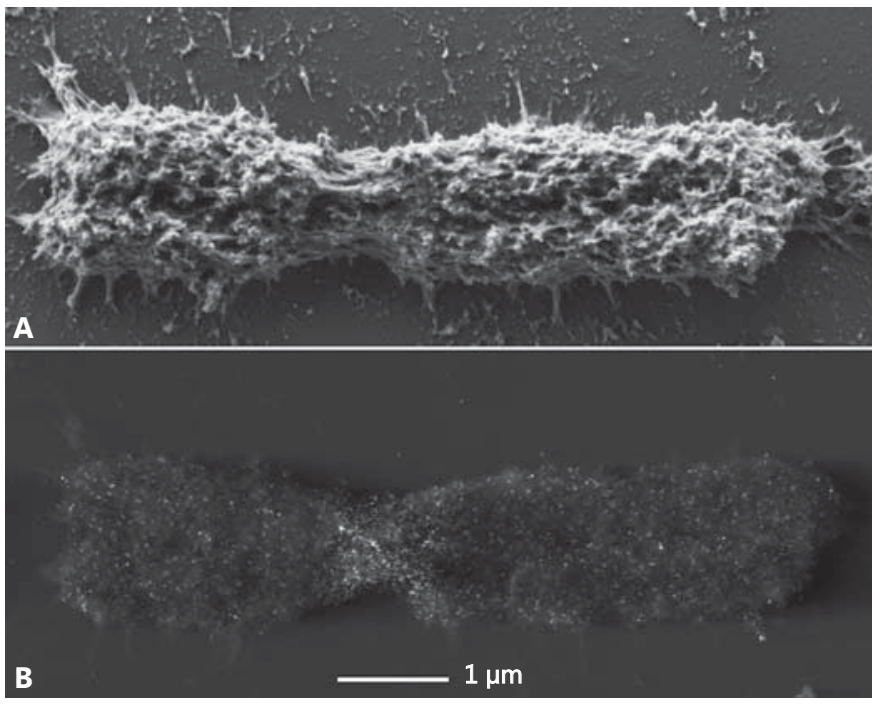

Fig. 3. Scanning electron micrographs of a topographic secondary electron image (A) and backscattered electron signal (B) of the anti-H2AThr120ph label distribution at the primary constriction of a single T. aestivum mitotic metaphase chromosome. The primary constriction is ultrastructurally characterized by exposed parallel fibrils (A). H2AThr120ph exhibits a high density of labels (white dots) in the primary constriction (B).
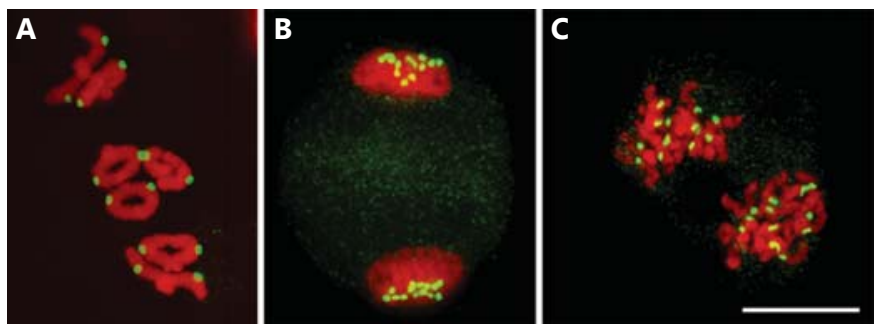

Fig. 4. Immunolabeling of meiotic $S$. cereale cells at A metaphase I, B interkineses, and C metaphase II with anti-H2AThr120ph (yellow). The size bar represents $10 \mu \mathrm{m}$.

To evaluate whether species, which were categorized earlier as holocentrics, possess indeed holocentric chromosomes, we immunostained mitotic metaphase cells of C. alternifolius and of different Drosera species with antiH2AThr120ph. Shirakawa et al. [2011] postulated the occurrence of mono- (D. regia) and holocentric (D. arcturi) chromosomes in Drosera due to presence or absence of a primary constriction at mitotic metaphase, respectively. As expected for Cyperus based on early electron microscopic studies by Braselton [1971], chromosomes of $C$. alternifolius displayed a holocentric-like distribution of 
immunofluorescence signals (fig. 1Q). In contrast, all Drosera species analyzed displayed a typical monocentric-like distribution of anti-H2AThr120ph immunosignals (fig. 1R-V). This result was unexpected for Drosera species, since previous studies indicated the occurrence of holocentric chromosomes in the genus based on their chromosome morphology (no primary constriction evident), the mitotic anaphase behavior (chromatids move as parallel linear bars), and the stable transmission of chromosome fragments [Kondo and Lavarack, 1984; Furuita and Kondo, 1990; Sheikh and Kondo, 1995; Sheikh et al., 1995; Hoshi, 2002]. Hence, it is likely that at least the analyzed species D. binata, D. alicia, and D. rotundi- folia possess monocentric chromosomes and not holocentric ones.

In summary, we have identified the first universal antibody-based marker which can be used to visualize centromeric chromatin of mono- and holocentric plant species.

\section{Acknowledgements}

A.H. was supported by the DFG Germany (SFB 648, SPP 1384) and by the German Federal Ministry of Education and Research (FKZ 0315965). The excellent technical assistance of J. Grünert is gratefully acknowledged.

\section{References}

Betts M, Russell R: Amino acid properties and consequences of substitutions, in Barnes MR (ed): Bioinformatics for Geneticists: A Bioinformatics Primer for the Analysis of Genetic Data, ed 2, pp 311-340 (John Wiley \& Sons Ltd, Hoboken 2007).

-Blower MD, Sullivan BA, Karpen GH: Conserved organization of centromeric chromatin in flies and humans. Dev Cell 2:319-330 (2002).

Braselton JP: The ultrastructure of the non-localized kinetochores of Luzula and Cyperus. Chromosoma 36:89-99 (1971).

-Dawe RK, Reed LM, Yu HG, Muszynski MG, Hiatt EN: A maize homolog of mammalian CENPC is a constitutive component of the inner kinetochore. Plant Cell 11:1227-1238 (1999).

Dong Q, Han F: Phosphorylation of histone H2A is associated with centromere function and maintenance in meiosis. Plant J 71:800-809 (2012).

Furuita T, Kondo K: Effects of $\gamma$-rays of diffusedcentromeric chromosomes of Drosera falconerii in vitro. Chrom Sci 3:93-100 (1990).

- Gendrel AV, Lippman Z, Martienssen R, Colot V: Profiling histone modification patterns in plants using genomic tiling microarrays. Nat Methods 2:213-218 (2005).

Gernand D, Demidov D, Houben A: The temporal and spatial pattern of histone $\mathrm{H} 3$ phosphorylation at serine 28 and serine 10 is similar in plants but differs between mono- and polycentric chromosomes. Cytogenet $\mathrm{Ge}$ nome Res 101:172-176 (2003).

-Gutierrez-Caballero C, Cebollero LR, Pendas AM: Shugoshins: from protectors of cohesion to versatile adaptors at the centromere Trends Genet 28:351-360 (2012).

-Han YH, Wang GX, Liu Z, Liu JH, Yue W, et al: Divergence in centromere structure distinguishes related genomes in Coix lacryma-jobi and its wild relative. Chromosoma 119:89-98 (2010).
Heckmann S, Schroeder-Reiter E, Kumke K, Ma L, Nagaki K, et al: Holocentric chromosomes of Luzula elegans are characterized by a longitudinal centromere groove, chromosome bending, and a terminal nucleolus organizer region. Cytogenet Genome Res 134:220-228 (2011).

Hoshi Y: Chromosome studies in Drosera (Droseraceae). Proc 4th Intl Carniv Pl Conf:31-38 (2002).

Houben A, Schubert I: DNA and proteins of plant centromeres. Curr Opin Plant Biol 6:554-560 (2003).

Houben A, Guttenbach M, Kress W, Pich U, Schubert I, Schmid M: Immunostaining and interphase arrangement of field bean kinetochores. Chromosome Res 3:27-31 (1995).

Houben A, Wako T, Furushima-Shimogawara R, Presting G, Künzel G, et al: The cell cycle dependent phosphorylation of histone $\mathrm{H} 3$ is correlated with the condensation of plant mitotic chromosomes. Plant J 18:675-679 (1999).

Houben A, Schroeder-Reiter E, Nagaki K, Nasuda $S$, Wanner G, et al: CENH3 interacts with the centromeric retrotransposon cereba and GCrich satellites and locates to centromeric substructures in barley. Chromosoma 116:275283 (2007).

Houben A, Kumke K, Nagaki K, Hause G: CENH3 distribution and differential chromatin modifications during pollen development in rye (Secale cereale L.). Chromosome Res 19:471480 (2011).

Jin WW, Melo JR, Nagaki K, Talbert PB, Henikoff S, et al: Maize centromeres: organization and functional adaptation in the genetic background of oat. Plant Cell 16:571-581 (2004).

Kawashima SA, Yamagishi Y, Honda T, Ishiguro K, Watanabe Y: Phosphorylation of H2A by Bub1 prevents chromosomal instability through localizing Shugoshin. Science 327: 172-177 (2010).
Kondo K, Lavarack PS: A cytotaxonomic study of some Australian species of Drosera 1 (Droseraceae). Bot J Linn Soc 88:317-333 (1984).

Liu Z, Yue W, Li DY, Wang RR, Kong XY, et al: Structure and dynamics of retrotransposons at wheat centromeres and pericentromeres. Chromosoma 117:445-456 (2008).

- Manzanero S, Arana P, Puertas MJ, Houben A: The chromosomal distribution of phosphorylated histone $\mathrm{H} 3$ differs between plants and animals at meiosis. Chromosoma 109:308317 (2000).

Mole-Bajer J, Bajer AS, Zinkowski RP, Balczon RD, Brinkley BR: Autoantibodies from a patient with scleroderma CREST recognized kinetochores of the higher plant Haemanthus. Proc Natl Acad Sci USA 87:3599-3603 (1990).

Moroi Y, Peebles C, Fritzler MJ, Steigerwald J, Tan EM: Autoantibody to centromere (kinetochore) in scleroderma sera. Proc Natl Acad Sci USA 77:1627-1631 (1980).

Nagaki K, Murata M: Characterization of CENH3 and centromere-associated DNA sequences in sugarcane. Chromosome Res 13:195-203 (2005).

Nagaki K, Cheng Z, Ouyang S, Talbert PB, Kim $M$, et al: Sequencing of a rice centromere uncovers active genes. Nat Genet 36:138-145 (2004).

Nagaki K, Kashihara K, Murata M: Visualization of diffuse centromeres with centromere-specific histone $\mathrm{H} 3$ in the holocentric plant $\mathrm{Lu}$ zula nivea. Plant Cell 17:1886-1893 (2005).

- Nasuda S, Hudakova S, Schubert I, Houben A, Endo TR: Stable barley chromosomes without centromeric repeats. Proc Natl Acad Sci USA 102:9842-9847 (2005)

- Sanei M, Pickering R, Kumke K, Nasuda S, Houben A: Loss of centromeric histone $\mathrm{H} 3$ (CENH3) from centromeres precedes uniparental chromosome elimination in interspecific barley hybrids. Proc Natl Acad Sci USA 108:E498-505 (2011). 
Schroeder-Reiter E, Houben A, Wanner G: Immunogold labeling of chromosomes for scanning electron microscopy: a closer look at phosphorylated histone $\mathrm{H} 3$ in mitotic metaphase chromosomes of Hordeum vulgare. Chromosome Res 11:585-596 (2003).

-Schubert I, Dolezel J, Houben A, Scherthan H, Wanner G: Refined examination of plant metaphase chromosome structure at different levels made feasible by new isolation methods. Chromosoma 102:96-101 (1993).

-Schubert V: No neocentric activity on Aegilops markgrafii chromosomes. Cytogenet Genome Res 132:100-104 (2010).

-Sheikh SA, Kondo K: Differential staining with Orcein, Giemsa, CMA, and DAPI for comparative chromosome study of 12 species of Australian Drosera (Droseraceae). Am J Bot 82:1278-1286 (1995).
Sheikh SA, Kondo K, Hoshi Y: Study on diffused centromeric nature of Drosera chromosomes. Cytologia 60:43-47 (1995).

Shirakawa J, Nagano K, Hoshi Y: A chromosome study of two centromere differentiating Drosera species, $D$. arcturi and D. regia. Caryologia 64:453-463 (2011).

Talbert PB, Masuelli R, Tyagi AP, Comai L, Henikoff S: Centromeric localization and adaptive evolution of an Arabidopsis histone H3 variant. Plant Cell 14:1053-1066 (2002).

ten Hoopen R, Manteuffel R, Dolezel J, Malysheva L, Schubert I: Evolutionary conservation of kinetochore protein sequences in plants. Chromosoma 109:482-489 (2000).
Wang F, Ulyanova NP, van der Waal MS, Patnaik D, Lens SM, Higgins JM: A positive feedback loop involving Haspin and Aurora B promotes CPC accumulation at centromeres in mitosis. Curr Biol 21:1061-1069 (2011).

Yu HG, Dawe RK: Functional redundancy in the maize meiotic kinetochore. J Cell Biol 151: 131-142 (2000).

Zhang H, Dawe RK: Total centromere size and genome size are strongly correlated in ten grass species. Chromosome Res 20:403-412 (2012).

Zhang WL, Friebe B, Gill BS, Jiang JM: Centromere inactivation and epigenetic modifications of a plant chromosome with three functional centromeres. Chromosoma 119:553563 (2010). 